\title{
Lumbar subdural cerebrospinal fluid collection with acute cauda equina syndrome after posterior fossa decompression for Chiari malformation Type I: case report
}

\author{
Houssein A. Darwish, MD, and Edward H. Oldfield, MD \\ Department of Neurological Surgery, University of Virginia Health System, Charlottesville, Virginia
}

\begin{abstract}
This report describes the circumstances of a patient with a cauda equina syndrome due to the development of a lumbar subdural CSF collection with ventral displacement of the cauda equina shortly following posterior fossa decompression for Chiari malformation Type I (CM-I). This unusual, but clinically significant, complication was successfully treated with percutaneous drainage of the extraarachnoid CSF collection. Although there are a few cases of intracranial subdural hygroma developing after surgery for $\mathrm{CM}-\mathrm{I}$, often attributed to a pinhole opening in the arachnoid, as far as the authors can determine, a spinal subdural hygroma associated with surgery for CM-I has not been recognized.
\end{abstract}

http://thejns.org/doi/abs/10.3171/2016.2.SPINE151480

KEY WORDS cauda equina; lumbar subdural CSF collection; Chiari malformation Type l; suboccipital craniectomy; congenital; deformity

I $\mathrm{N}$ this report we summarize the clinical events occurring shortly after surgery for a Chiari malformation Type I (CM-I) and associated with the development of a cauda equina syndrome as a result of a nonhemorrhagic, subdural, extraarachnoidal CSF collection. A few cases of intracranial subdural hygroma following surgery for a CM-I have been reported.$^{1-4,6,7}$ However, a symptomproducing spinal subdural hygroma associated with decompressive surgery for CM-I has not been recognized. This clinically significant complication was successfully treated with percutaneous drainage of the extraarachnoid CSF collection.

\section{Case Report}

History and Examination

A 40-year-old female presented with intractable suboccipital cough headaches. Her clinical evaluation demonstrated a normal neurological examination, and MRI of her cervical spine showed a typical CM-I without associated syringomyelia (Fig. 1). Posterior fossa decompression was performed with removal of the posterior margin of the foramen magnum, C-1 laminectomy, opening the dura while attempting to keep the arachnoid intact, and dural grafting with autologous pericranium. While opening the dura, a pinhole breach (1-2 $\mathrm{mm})$ in the arachnoid occurred. Initially, pulses of CSF passing through it were obvious with each heartbeat, but these subsided completely after a brief interval of drainage. Thus, no attempt was made to repair the pinhole defect. On the day following surgery, the patient complained of pain in her lower back extending down the back of her thighs, occasionally to the level of her ankles, with intermittent numbness. However, her neurological examination remained normal. She was discharged on postoperative Day 2 with resolution of her headaches but persistent pain in her lower back and posterior thighs.

Six days after discharge, she returned to the clinic with severe back pain radiating into both lower extremities. The previous night she had noticed difficulty with urination, particularly on initiating urination, but she did not have incontinence or saddle anesthesia; she also had headaches, although her neurological examination was normal, including her sphincter tone. Magnetic resonance imaging of the cervical, thoracic, and lumbar spine showed the conus medullaris, cauda equina, and filum terminale displaced anteriorly, with the cauda equina plastered ventrally against the dura adherent to the posterior wall of the 


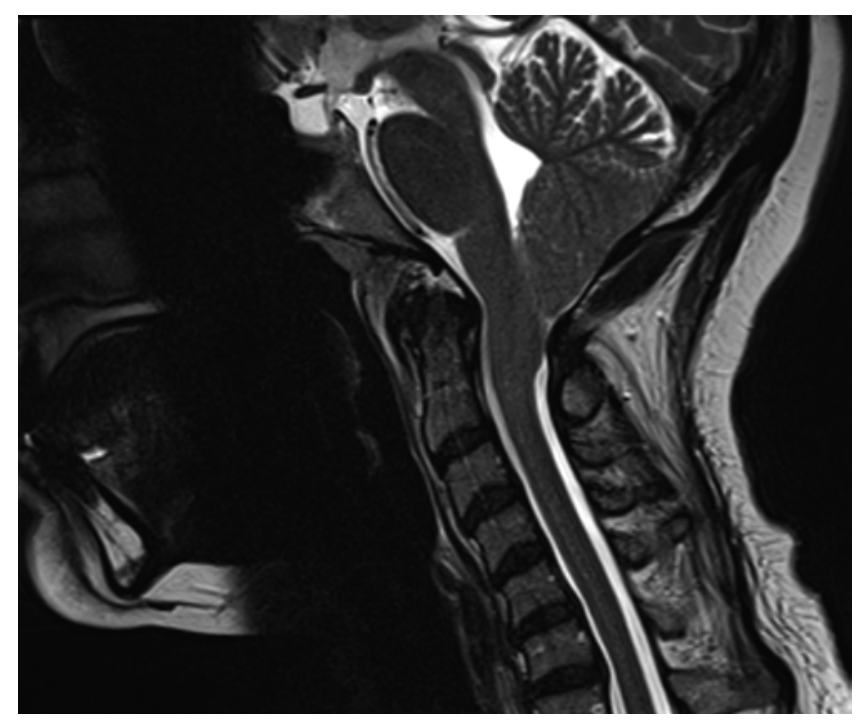

FIG. 1. Preoperative sagittal cervical T2-weighted MR image showing CM-I with a pointed shape of the cerebellar tonsils, the tips of which are at the level of C-1. Note the absence of subdural fluid above the cerebellum.

vertebral bodies (Fig. 2C and D). On the axial views there was a dorsal subdural collection with signal identical to that for CSF on T1- and T2-weighted imaging, compressing the intraarachnoidal structures anteriorly. The thoracic spine T2-weighted MRI showed CSF-like fluid posteriorly in the subdural space (Fig. 2B). The cervical T2-weighted MRI showed an extraarachnoid subdural collection of fluid along the superior margin of the cerebellum with mild mass effect (Fig. 2A). The collection of subdural extraarachnoidal fluid with CSF-like signal appeared to communicate between the lumbar and thoracic subdural spaces.

\section{Treatment}

The patient was admitted to the hospital, a lumbar drain was inserted at the L4-5 interlaminar space, and under fluoroscopic control the tip was placed into the lumbar subdural space where it drained the CSF-like fluid. After passively draining $40 \mathrm{ml}$, the drain was left in place with the patient at bedrest for 48 hours. Her low-back and left leg pain resolved after 24 hours of CSF drainage. She still had residual pain in her right lower extremity, although it was substantially improved. After the drain was removed at 48 hours, she was out of bed and ambulating with only mild residual low-back pain. Repeat MRI demonstrated the disappearance of the ventral displacement of the cauda equina.

\section{Posttreatment Course}

Two months later she returned to the clinic, when MRI showed resolution of the cerebellar tonsil herniation with the tonsils' return to a normal shape and position, resolution of the subdural collection above the cerebellum and in the thoracic region, disappearance of the lumbar subdural CSF collection, and a normal position of the cauda equina (Fig. 3). At that visit she still had pain in her right leg, exacerbated by coughing and laughing. When seen in the clinic 8 months later, she described continuing spo-
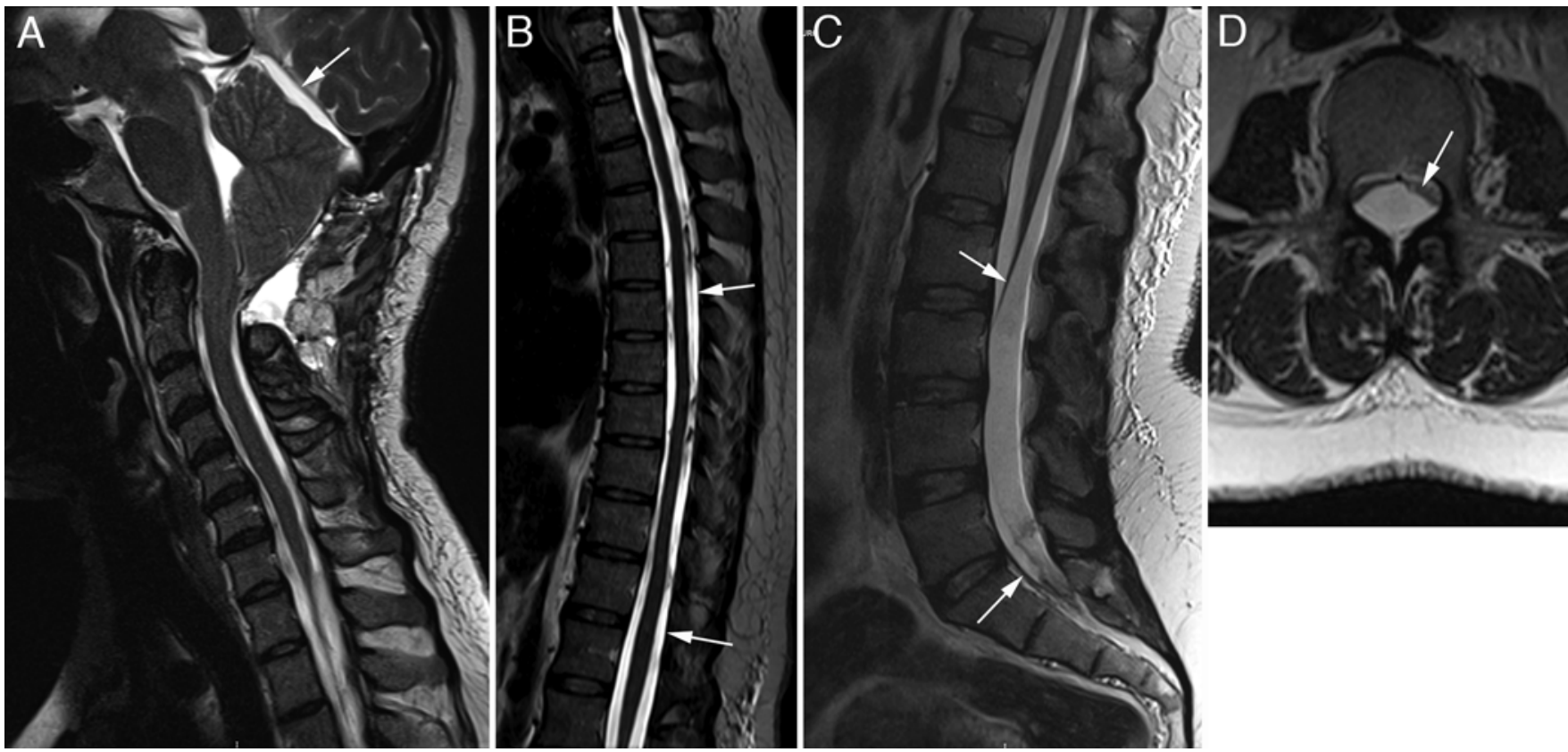

FIG. 2. Magnetic resonance images obtained 7 days after surgery. A: Sagittal T2-weighted image demonstrating the subdural, extraarachnoid CSF collection (arrow) between the tentorium and the superior margin of the cerebellum with mild mass effect. B: Sagittal thoracic spine T2-weighted MR image showing the subdural CSF collection (arrows) along the posterior margin of the spinal canal. C and D: Sagittal and axial lumbar spine T2-weighted MR images. Note the conus medullaris and cauda equina anteriorly displaced (arrows) by the subdural extraarachnoidal collection, which has a signal identical to CSF. 

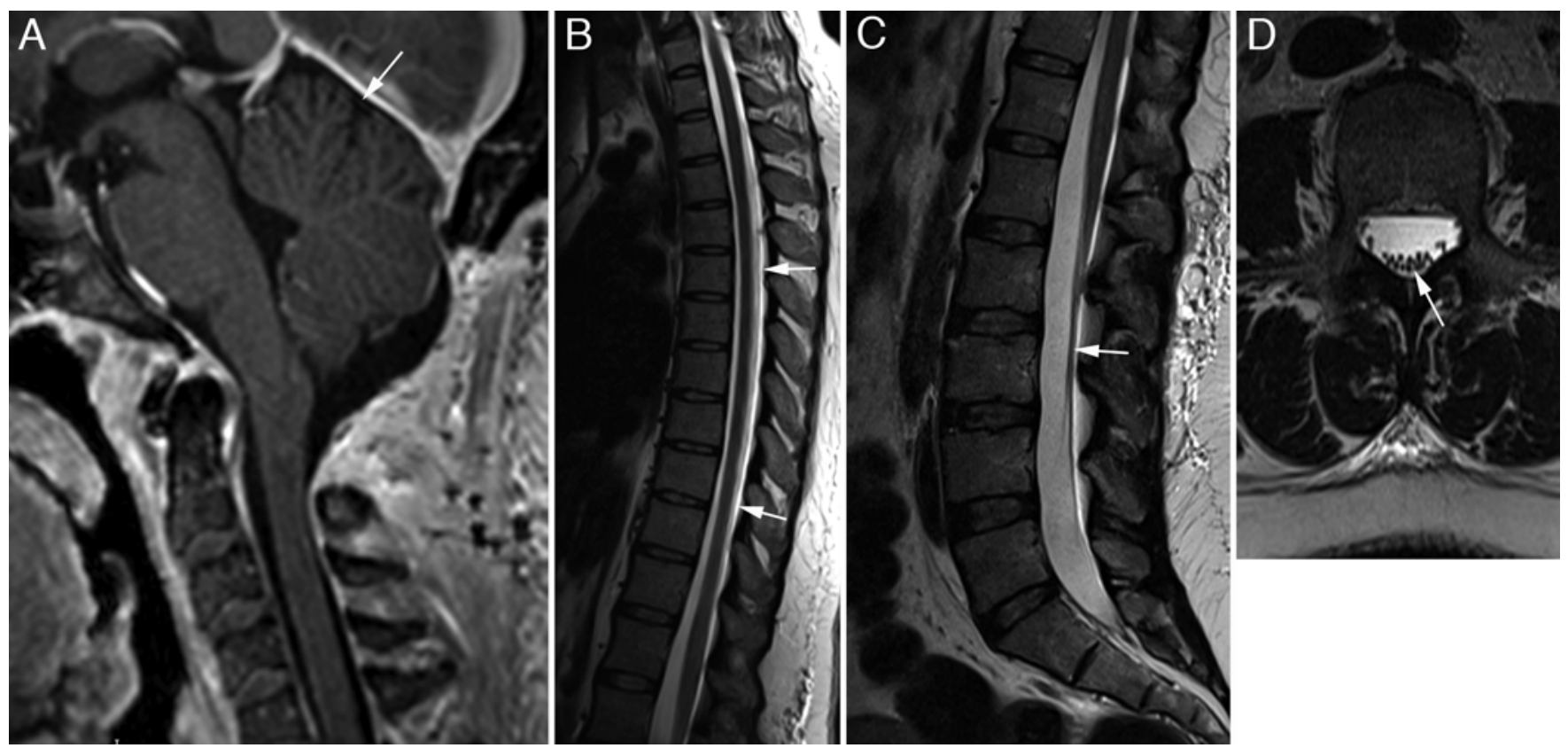

FIG. 3. Magnetic resonance images obtained at the 2-month follow-up. A: Sagittal cervical MR image showing the resolution of tonsillar herniation, return of the tonsils to a normal shape, and disappearance of the extraarachnoid CSF collection superior to the cerebellum. B: Sagittal thoracic MR image showing nearly complete resolution of the thoracic subdural CSF collection. C and D: Sagittal and axial lumbar MR images. The subdural CSF collection is gone, and the conus medullaris and cauda equina are now in a normal position. Similar brain and lumbar MRI was performed 6 months later.

radic pain and numbness in her right lower extremity that was exacerbated by bending forward and disturbed her sleep. Her neurological examination, including straight leg raising, was negative, and brain and lumbar spine MRI showed no evidence of hygroma recurrence.

\section{Discussion}

Several cases of a subdural hygroma under pressure developing in the posterior fossa or the supratentorial space have been reported. ${ }^{1-4,6,7}$ The patients generally developed symptoms 3-15 days after the surgery. In all of them the original surgery included opening the dura, and in many of them an attempt was made to leave the arachnoid intact and a small pinhole leak in the arachnoid was noted at surgery. Some leaks were associated with an extradural collection of CSF under pressure at the wound site with CSF leakage. In different circumstances, Singleton et al. recently reported on 2 patients who developed subdural hygromas in the lumbar spine with ventral compression of the cauda equina, both with a cauda equina syndrome associated with an inadvertent durotomy during spinal disc surgery. ${ }^{5}$ Both patients had surgical exploration with drainage of a tense subdural hygroma, and in both instances a wide opening of the arachnoid was created without later recurrence of the hygroma.

Although our patient underwent suboccipital craniectomy and C-1 laminectomy, the symptom-producing subdural CSF collection under pressure occurred in the lumbar region. Singleton and colleagues described 2 patients with postoperative subdural, extraarachnoidal CSF collections in the lumbar region following lumbar decompression sur- gery via laminectomy complicated by durotomy. ${ }^{5}$ In both patients the symptoms of a cauda equina syndrome started 24 hours after the surgery. Magnetic resonance imaging in both patients showed a subdural, extraarachnoidal CSF collection displacing the cauda equina ventrally. The mechanism proposed by the authors was a ball-valve oneway flow of CSF through a pinhole defect in the arachnoid with CSF collecting beneath the dura under pressure. Similar observations have been made in several patients with intracranial subdural hygromas developing after craniovertebral decompression for CM-I. The long-standing practice of one of us (E.H.O.) has been to attempt to open the dura while leaving the arachnoid intact, to keep blood out of the subarachnoid space and reduce the risk of arachnoid adhesions developing later. However, it is not always possible to open the dura while keeping the arachnoid intact. Our patient also had pulses of CSF passing through a pinhole defect in the arachnoid, which was noted as the dura was being opened. By the time the pericranial graft was acquired and placed, the CSF had drained somewhat and the pulses were no longer obvious. Thus, no attempt was made to either close the defect in the arachnoid or enlarge it.

Thus, in our patient the pinhole arachnoidal tear created a ball-valve one-way efflux of CSF flow driven by the systolic pulses in the CSF space. This assertion is supported by the presence of the thoracic subdural collection communicating with the lumbar extraarachnoidal collection. This could have probably been prevented by enlarging the opening in the arachnoid. It was promptly and successfully treated, not by further surgery to address the arachnoid leak in the region of the foramen magnum, but 
with a percutaneous lumbar drain with its tip in the subdural collection, which suggests that the pinhole leak in the arachnoid at the foramen magnum either closed spontaneously or that drainage of the lumbar hygroma somehow facilitated its closure.

\section{Conclusions}

A conventional operation for a CM-I with dural opening and placement of a pericranial graft was complicated by the cauda equina syndrome associated with a large extraarachnoid, subdural CSF collection in the lumbar region. We probably could have prevented this by enlarging the arachnoid opening and thus preventing the ball-valve one-way CSF flow. The circumstances in this patient demonstrate the importance of considering a spinal subdural fluid collection if a patient develops a cauda equina syndrome after surgery for a CM-I.

\section{References}

1. Bahuleyan B, Menon G, Hariharan E, Sharma M, Nair S: Symptomatic posterior fossa and supratentorial subdural hygromas as a rare complication following foramen magnum decompression for Chiari malformation Type I. J Neurosurg 114:510-513, 2011

2. Filis AK, Moon K, Cohen AR: Symptomatic subdural hygroma and hydrocephalus following Chiari I decompression. Pediatr Neurosurg 45:425-428, 2009

3. Pereira EAC, Steele LF, Magdum SA: Recurrent subdural hygromas after foramen magnum decompression for Chiari Type I malformation. Br J Neurosurg 28:396-399, 2014

4. Ranjan A, Cast IP: Symptomatic subdural hygroma as a complication of foramen magnum decompression for hind- brain herniation (Arnold-Chiari deformity). Br J Neurosurg 10:301-303, 1996

5. Singleton WGB, Ramnarine D, Patel N, Wigfield C: Postoperative spinal subdural extra-arachnoid hygroma causing cauda equina compression: a report of two cases. Br J Neurosurg 26:429-431, 2012

6. Suzuki F, Kitagawa T, Takagi K, Nozaki K: Subacute subdural hygroma and presyrinx formation after foramen magnum decompression with duraplasty for Chiari type 1 malformation. Neurol Med Chir (Tokyo) 51:389-393, 2011

7. Zakaria R, Kandasamy J, Khan Y, Jenkinson MD, Hall SR, Brodbelt A, et al: Raised intracranial pressure and hydrocephalus following hindbrain decompression for Chiari I malformation: a case series and review of the literature. Br J Neurosurg 26:476-481, 2012

\section{Disclosures}

The authors report no conflict of interest concerning the materials or methods used in this study or the findings specified in this paper.

\section{Author Contributions}

Conception and design: both authors. Acquisition of data: both authors. Analysis and interpretation of data: both authors. Drafting the article: both authors. Critically revising the article: both authors. Reviewed submitted version of manuscript: both authors. Approved the final version of the manuscript on behalf of both authors: Oldfield. Study supervision: Oldfield.

\section{Correspondence}

Edward H. Oldfield, Department of Neurological Surgery, University of Virginia, Box 800212, Charlottesville, VA 22908. email: eho4u@virginia.edu. 